\title{
Gold nanoparticle fluorescent molecular beacon for low-resolution DQ2 gene HLA typing.
}

\author{
Valerio Beni, Taye Zewdu, Hamdi Joda, Ioanis Katakis and Ciara K. O’Sullivan
}

\section{Linköping University Post Print}

N.B.: When citing this work, cite the original article.

The original publication is available at www.springerlink.com:

Valerio Beni, Taye Zewdu, Hamdi Joda, Ioanis Katakis and Ciara K. O’Sullivan, Gold nanoparticle fluorescent molecular beacon for low-resolution DQ2 gene HLA typing., 2012, Analytical and Bioanalytical Chemistry, (402), 3, 1001-1009.

http://dx.doi.org/10.1007/s00216-011-5493-2

Copyright: Springer Verlag (Germany)

http://www.springerlink.com/?MUD=MP

Postprint available at: Linköping University Electronic Press

http://urn.kb.se/resolve?urn=urn:nbn:se:liu:diva-86353 


\title{
Gold Nanoparticle Fluorescent Molecular Beacon for Low Resolution DQ2 HLA Typing
}

\author{
Valerio Beni $^{1^{*}}$, Taye Zewdu ${ }^{1}$, Hamdi Joda ${ }^{1}$ Ioanis Katakis $^{1}$ and Ciara K.O'Sullivan ${ }^{1,2^{*}}$ \\ ${ }^{I}$ Nanobiotechnology and Bioanalysis Group, Departament d'Enginyeria Quimica, Universitat Rovira i Virgili, \\ Av. Pasos Catalans, 26, 43007, Tarragona, Spain \\ ${ }^{2}$ Institucio Catalana de Recerca i Estudis Avanats, Passeig Lluis Companys 23, 08010, Barcelona, Spain
} Corresponding authors e-mail: ciara.osullivan@urv.cat and valerio.beni@urv.cat

Tel: $+34977558576 \quad$ Fax: +34977559621

\begin{abstract}
Coeliac disease is an inflammation of the small intestine triggered by gluten ingestion. Herein we present a fluorescent genosensor, exploiting molecular beacons functionalised gold nanoparticle, for the identification of Human Leukocyte Antigens (HLA) DQ2 gene, a key genetic factor in coeliac disease.

Optimisation of sensor performance was achieved by tuning the composition of the oligonucleotide monolayer immobilised on the Au nanoparticle and the molecular beacon design.

Co-immobilisation of the molecular beacon with a spacing oligo (thiolated 10-Thymines oligo) in the presence of 10 Adenines oligos, resulted in a significant increase of sensor response due to improved spacing of the molecular beacons and extension from the nanoparticle surface, which renders them more available for recognition.

Further increase in response (ca. $40 \%$ ) was shown to be achievable when the recognition sequence of the molecular beacon was incorporated in the stem.

Improvement of the molecular beacons' specificity was also achieved by the incorporation within their recognition sequence of a one-base mismatch. Finally, a gold nanoparticle functionalised with two molecular beacons targeting the DQA $1 * 05 *$ and DQB $1 * 02 *$ alleles has been demonstrated to allow the low resolution typing of the DQ2 gene at nanomolar level.
\end{abstract}

Keywords: gold nanoparticles, HLA Typing, molecular beacons, fluorescence genosensor, coeliac disease,

\section{Introduction}

Coeliac disease (CD), a genetically and environmentally induced inflammation of the small intestine (1), is promoted by the ingestion of gluten, a protein present in wheat, barley, and rye (2). This disease has been associated with nutrient malabsorption, growth problems, gastrointestinal disorders and 
increased risks of osteoporosis, infertility, autoimmune diseases, and lymphomas (2). Symptomatic diagnosis of CD is quite difficult and currently the golden standard for its diagnosis is biopsy of the small intestine. Recently, genetic testing (HLA typing) has been proposed as a tool to evaluate the predisposition to $\mathrm{CD}$ (3-5), because almost $100 \%$ of affected patients carry, at least, one of the two genotypes, DQ2 cis and trans, (ca. 95\%) or DQ8 (ca. 5\%). These genotypes are coded by the following combination of alleles: DQA1*0501/DQB1*0201 (DQ2-cis) or DQA1*0505/DQB1*0202 (DQ2-trans) and DQA1*0301/DQB1*0302 (DQ8) $(2,3,6)$.

In recent years genetic analysis has gained ground in fields as bio-diagnostics, environmental monitoring, food safety and quality control, forensics and bio-hazard investigations (7-9), resulting in a growing interest in DNA biosensors (genosensors). However, the fact that the physical changes occurring during the recognition event (hybridisation) are not easily measurable resulted in the development of highly sensitive transduction approaches based on electrochemical techniques $(10,11)$, and optical detection (12).

Since their introduction by Tyagi and Kramer (13), fluorescence molecular beacons (MBs), have been vastly explored (13-15). These are specifically engineered bi-functional (quencher and fluorophore) short oligonucleotide chain probes characterised by a stem-loop structure; this allows them to change their spatial conformation upon recognition of the DNA target and, as a result of this, to generate fluoresce (13). Originally in MBs quenching was provided by molecular quenchers (14) but since 2001 $\mathrm{Au}$ surfaces (16-18) and even more gold nanoparticles (AuNPs) (19-25) have found application as highly efficient and universal quenchers. Moreover the use of Au based quenching has been shown to be characterised by minimal background fluorescence and subsequently improved signal-tobackground ratio $(9,23,26)$.

The high quenching efficiency of AuNPs is due to the associated surface plasmon resonance and its ability to enhance all the radiative and non-radiative properties of the NPs (27). In the case of nanoparticles quenching has been demonstrated to be governed by the surface energy transfer (28) and to be strongly dependent on donor-acceptor distances (29).

Recently, various authors reported on the used of AuNP-DNA molecular beacons in the development of bioassays for genetic disease detection (19). Maxwell et al. (20) and Ray et al. (22, 23) described sensing platforms based on the use of fluorophore modified DNA sequence immobilised on AuNPs, taking advantage of the tendency of the ssDNA to organise itself into an "arch" structure when immobilised. In an other approach Mo et al.(21) reported an AuNPs fluorescence sensing platform based on the displacement of not fully complementary fluorophore modified DNA sequence by the fully complementary target. Zhang et al. (24) developed an interesting sensing platform based on a 
reverse molecular beacon design, where work a FAM modified linear probe, immobilised onto AuNPs assumed a blocked loop structure upon hybridisation with target, resulting in quenching of the FAM fluorescence. Finally Wang et al. (16) reported on the development of a platform for the detection of single nucleotide polymorphisms (SNP) based on the combination of AuNPs and ligase reaction. In this work the discrimination was based on the different abilities of ssDNA and dsDNA to absorb onto AuNPs and on the resulting changes in fluorescence according if the fluorescence labelled primers were in their single strand form (no ligation occurred) or as double strand (ligation occurred).

The first report on the use of AuNPs as quenchers in MB and their application for the detection of specific DNA sequence was presented by Dubertret et al. (19), where the authors demonstrated the possibility of replacing the quencher in a MB with AuNPs. Following this first report other authors investigated the use of MBs-AuNPs for the detection of genetic disease (12) demonstrating the ability to discriminate single point mismatches $(26,30)$. Significant improvement in the state-of-the-art was achieved by Song et al. (9) where the authors, by taking fully advantage of the quenching efficiency and scaffolding ability of AuNPs, demonstrated the possibility of having three perfectly and independently working molecular beacons immobilised on a single AuNPs highlighting the possibility of single particle multiplex detection of DNA markers.

In the work we report herein, the development and optimisation of an AuNP based fluorescence bioassay for the low resolution typing of the DQ2 CD associated genotype is presented. Key points investigated in this work were the design of the molecular beacons, the functionalisation of AuNPs as well as the composition of the oligonucleotide monolayer immobilised onto the AuNPs. The specificity of the developed beacons was evaluated and applied to the discrimination on single base mismatches. Furthermore, the AuNP-MB conjugate were characterised and the developed molecular beacon was used for the low resolution HLA typing of the DQ2-genotype.

\section{Experimental}

\subsection{Materials.}

All chemicals were used as purchased without further purification. Potassium dihydrogen phosphate, potassium monohydrogen phosphate were purchased from Fluka (Barcelona, Spain), sodium chloride, sodium hydroxide $2 \mathrm{~N}$, hydrochloric acid $6 \mathrm{~N}$ and concentrated nitric acid were purchased from Scharlau Chemie S.A. (Barcelona, Spain), sodium citrate, chloroauric acid, Zonyl FSN, dithiothreitol (DTT) and Tris carboxy ethyl phosphine (TCEP) were purchased from Sigma (Barcelona, Spain) and concentrated hydrochloric acid was obtained from Merck (Darmsted, Germany). Twenty x SSC (saline-sodium citrate buffer) was obtained from Sigma. The oligonucleotides were synthesised by 
www.biomers.net (Ulm, Germany) and were reconstituted to a $100 \mu \mathrm{M}$ stock solution using ultra pure water and stored at $-20{ }^{\circ} \mathrm{C}$. All solutions were prepared in high purity water obtained from a Milli-Q RG system (Barcelona, Spain). Hybridisation experiments were performed in $1 \mathrm{x}$ SSC buffer at pH 7.4. The sequences used in this work are listed in Table 1. The recognition sequences are underlined and the bases in italic represent the complementary strands of the stems.

\section{LOCATION TABLE 1}

\subsection{Design of Molecular Beacons}

In the reported work molecular beacons for low resolution DQ2 HLA typing were designed. In order to perform HLA typing of DQ2 genotype the detection of the DQA $1 * 0501$, DQA $1 * 0505$, DQB $1 * 0201$ and DQB $1 * 0202$ alleles is required; a schematic representation of the alleles combination associated to the DQ2 gene, for both its possible forms (cis and trans), is reported in Scheme 1. In this Scheme the alleles universally accepted to be associated with $\mathrm{CD}$ are depicted in black, whilst the alleles encountered more frequently in combination with those associated to $\mathrm{CD}$ are depicted in white.

In HLA typing "low resolution" is defined as the detection of a family of alleles. In the reported work two molecular beacons for low resolution HLA typing of DQA $1 * 05 *$ and DQB $1 * 02 *$ allele's families were designed. The two probes, DQA $1 * 05 * \mathrm{MB}$ probes and $\mathrm{DQB} 1 * 02 * \mathrm{MB}$ probes, were designed to detect, respectively, DQA1*050101/DQA1*0505 and DQB1*0201/DQB1*0202 CD's associated alleles but not to discriminate between them. It must be notice that the two MB were also able to detect some other alleles: DQA $1 * 0508 / 0509 / 0510$ in the case of the DQA $1 * 05 *$ probe and the DQB $1 * 0204 / 0205 / 0206$ in the case of the DQB $1 * 02 *$ probe. These alleles, not associated to the CD disease, are rare having a prevalence below $1 \%$ (31) and for this are not expected to generate significant false positive.

\section{LOCATION SCHEME 1}

All MBs consisted of a 17-nucleotide long recognition sequence and a 5-nucleotide stem, with 10 thymines between the functional elements of the MB and a terminating thiol group. The MBs were modified at their 5' end with the fluorophore and at their 3' end with a thiol. Rhodamine 6G (adsorption $525 \mathrm{~nm}$, emission $555 \mathrm{~nm}$ ) and Atto 647N (adsorption $644 \mathrm{~nm}$, emission $669 \mathrm{~nm}$ ) were used as the fluorophores for the DQA $1 * 05 *$ and DQB $1 * 02 *$ MB probes, respectively (Figure S1). Two different designs of the molecular beacon differing for the location of the recognition were investigated. In the first design the recognition sequence was coinciding with the loop of the $\mathrm{MB}$; in order to achieve this two 5 bases long sequences (stem), one reverse complementary of the other, were added at each end of the recognition sequence $(9,12-14)$. In the second design, the recognition sequence was partially 
incorporated in the stem (32); in this design 5 nucleotide long appendage, complementary to the last 5 bases of the 3' end of the recognition sequence, were added between the 5' end of the loop and the fluorophore. This second design was anticipated to introduce increased stringency and consequently an improved specificity $(32,33)$; moreover it was also envisaged that this design, upon hybridisation, will result in an increased rigidity of the formed duplex, efficiently extending the fluorophore far from the AuNPs, providing a higher fluorescent signal.

In order to improve the selectivity of the MB probes the use of amplification refractory mutation system, ARMS (25) type approach for the design of the recognition sequences was also investigated. This approach introduces an artificial mismatch in the recognition part of the MB with the aim of improving the selectivity for fully complementary over single nucleotide mismatch as the duplex of the MB with the mismatched sequence would be less stable than the full matched sequence.

\subsection{Fluorescence measurements}

All fluorescence measurements were performed using black quartz cuvettes with $1 \mathrm{~cm}$ path length and with a total maximum volume of $150 \mu \mathrm{L}$. The excitation and emission wavelength used were 525 or $555 \mathrm{~nm}$ and 644 or $669 \mathrm{~nm}$, for the Rhodamine 6G and the Atto 647N respectively. Slits of $5 \mathrm{~nm}$ were used for both excitation and emission. All the experiments were performed using a photomultipler tube voltage of $600 \mathrm{~V}$. All measurements were performed at controlled temperature $\left(25^{\circ} \mathrm{C}\right)$.

Molecular beacon response was reported as the relative increase of the fluorescence intensity $\left(R_{f}\right)$, which was calculated as:

$$
R_{f}=\left(I_{\text {target }}-I_{\text {blank }}\right) /\left(I_{\text {probe }}-I_{\text {blank }}\right),
$$

where:

$I_{\text {probe }}$ fluorescence intensity before hybridization,

$I_{\text {target }}$ the fluorescence intensity after hybridization with target sequences,

$I_{\text {blank }}$ is the fluorescence intensity of background (bare AuNP in detection buffer).

Hybridization efficiency $\left(H_{e}\right)$ was calculated as:

$$
H_{e}=\left(I_{\text {target }}-I_{\text {probe }}\right) /\left(I_{D T T}-I_{\text {probe }}\right) \times 100
$$

where:

$I_{D T T}$ fluorescence intensity after Au-S bond cleavage by $0.1 \mathrm{M}$ DTT and removal of quencher AuNPs from solution.

The discrimination ability of MBs was expressed as the discrimination factor $(D f)$, 
$D f=\left(I_{\text {positive }}-I_{\text {blank }}\right) /\left(I_{\text {negative }}-I_{\text {blank }}\right)$

where:

$I_{\text {positive }}$ fluorescence intensity upon addition of the complementary target.

$I_{\text {negative }}$ fluorescence intensity upon addition of the non-complementary target.

\subsection{AuNPs synthesis and functionalisation}

AuNPs with average diameters of $15 \mathrm{~nm}$ were prepared via hot reduction of $\mathrm{NaAuCl}_{4}$ solution with sodium citrate $(34,35)$. Functionalisation of the AuNPs with the MBs was performed using in the presence of Zonyl FSN as it has been shown to provide much higher stabilisation of the AuNPs even at high concentrations $\mathrm{Na}^{+}(35,36)$. Briefly, an aliquot of freshly cleaved oligonucleotides /using TCEP) was added to a suspension of the AuNPs $(2.5 \mathrm{nM})$ in $10 \mathrm{mM}$ phosphate buffer containing Zonyl FSN $(0.05 \%, \mathrm{v} / \mathrm{v})$ at $\mathrm{pH} 7.4$ to obtain a final DNA/AuNPs ration of 500 to 1 (12) and left to react, at room temperature, for 20 minutes under shaking conditions (1400g). The MB/AuNPs mixture solution was then subjected to a so called "ageing process" consisting in a stepwise increase of the concentration of $\mathrm{NaCl}$ (steps of $0.1 \mathrm{M}$ ) up to $0.7 \mathrm{M}$, prior to an overnight incubation at $4{ }^{\circ} \mathrm{C}$. The $\mathrm{MB}$ functionalised AuNPs were then concentrated by centrifugation (14000 rpm, $20 \mathrm{~min}, 10^{\circ} \mathrm{C}$ ), washed, by a sequence of centrifugation/resuspension steps, and finally resuspended in the measurement buffer.

The immobilisation of the MBs was visualised using TEM imaging, and quantitatively evaluated by monitoring the fluorescence of the AuNP-MB conjugate following cleavage of the S-Au bond with dithiothreitol, DTT. Finally melting curve analysis was used to confirm stability of the AuNP-MB conjugate and to evaluate the ability of the MBs to open.

\section{Results and Discussion}

The envisaged mechanism of the proposed sensing platform is presented in Scheme 2. As typical with molecular beacons, in the absence of target DNA no significant fluorescence is recorded as the MB is in the closed configuration resulting in fluororescence quenched by the AuNPs). Upon hybridization of the immobilized MBs with the target sequences, an increase in fluorescence emission (flourophores moved away from AuNPs) is observed.

\section{LOCATION OF SCHEME 2}

\subsection{Selection of fluorophores}


Due to the strong quenching efficiency of AuNPs, a wide range of fluorophores could be used (14); the fluorophores chosen in this work were Atto $647 \mathrm{~N}$ and Rhodamine $6 \mathrm{G}$, as they are $\mathrm{pH}$ insensitive, relatively photostable and their absorption/emission spectra are well resolved (emission maxima at 669 and $555 \mathrm{~nm}$, respectively). In order to calculate the nanoparticle loading of the MBs, calibration curves between 1 and $1000 \mathrm{nM}$ were performed, for both the fluorophore modified oligos and a good linear response was obtained, Atto $647 \mathrm{~N}\left(\mathrm{Y}=0.94 \mathrm{X}+9.67 ; \mathrm{R}^{2}=0.993\right)$ Rhodamine $6 \mathrm{G}(\mathrm{Y}=0.62 \mathrm{X}+4.51$; $\left.\mathrm{R}^{2}=0.986\right)$.

\subsection{AuNPs and AuNP-MB conjugates characterization}

The size of the synthesised nanoparticles was confirmed using TEM measurements, and found to be 15 $\pm 2.6 \mathrm{~nm}(\mathrm{n}=100)$. UV-Vis measurement at $520 \mathrm{~nm}$ was used to calculate the concentration of the synthesised nanoparticles (ca. $4 \mathrm{nM}$ ); the extinction coefficient used in this measurement, $2.08 * 10^{8}$ $\mathrm{Lmol}^{-1} \mathrm{~cm}^{-1}$, was calculated assuming a spherical geometry of the nanoparticles and density equivalent to that of bulk gold (19.30 $\left.\mathrm{gcm}^{-3}\right)$ according to the approach proposed by Liu et al. (37). UV-VIS measurements were also used to check for the presence of aggregated particles (presence of a broad peak at ca. $700 \mathrm{~nm}$ ) as well as to confirm the presence of the DNA immobilised on the AuNPs, by measurement at $260 \mathrm{~nm}$ (12). Visualisation of DNA immobilised on the AuNPs was observed using TEM imaging (Figure 1); a clear difference in the capping layer thickness, from ca. $2 \mathrm{~nm}$ to 6-7 nm, prior to and following functionalisation with the molecular beacon, was observed. This difference in capping layer, upon functionalisation with DNA, has been reported previously by Stakenborg et al. (38); in the specificity of this work this was due to the displacement of the Zonyl by the molecular beacons. A quantification of the MBs immobilised on the AuNPs was performed via DTT cleavage experiments and was determined to be $41 \pm 7$ ( $n=3$ ) MBs for AuNPs.

\section{LOCATION OF FIGURE 1}

Finally thermal studies were performed to further confirm the presence of the MB onto the AuNPs and to demonstrate the level of fluorescence expected upon opening of the MBs (FigureS2). No significant variation in fluorescence was recorded with increasing temperatures until the melting temperature was reached, where a rapid increase in fluorescence, due to the opening of the MBs was observed.

\subsection{Design of molecular beacons}

The performances of the AuNPs-MB were expected to be depend not only on the design of the recognition element incorporated in the MB but also on the design of the MB itself (12). Two different designs of MB, differing in the position of the stem were thus evaluated. For the optimisation of the 
design a molecular beacon able to detect DQA1*0201 allele was designed; this choice was made because the sequence of a probe specific for this allele is well known. The two MBs (DQA1*0201a and DQA1*0201b from Table 1) were immobilised, in a singleplex format, onto AuNPs and the relative increase of the fluorescence upon hybridisation with $250 \mathrm{nM}$ of the fully complementary target (DQA $1 * 0201$ of Table 1), monitored. DTT cleavage experiments (Figure 2A) indicates that the use of such a design increased can increase the loading onto the AuNPs presumably due to the reduced loop size, with approximately $40 \%$ higher signal observed. The target hybridisation results are consistent with this increase in MB loading, with a $40 \%$ higher signal upon target binding also being observed although improved efficiency in MB opening can also contribute to the increase in response.

\section{LOCATION OF FIGURE 2}

\subsection{On-particle DNA monolayer composition}

The composition of the DNA monolayer immobilised onto the AuNPs has been observed to strongly influence the performance of AuNP-MB (9). In the work reported here the use of a poly-T spacer oligo and a complementary poly-A oligo was investigated to modulate the composition of the oligonucleotide layer, thus improving sensor performance. The use of spacer oligos length comparable to those of the MB-thiol poly-T appendage has been demonstrated to improve the efficiency of AuNPs immobilised-MBs (9). In this work spacer oligos (10 bases long thiol functionalised poly-T oligonucleotide) were used to space out the molecular beacons. To further improve the spacing, a 10base poly-A was used to hybridise to the poly $-\mathrm{T}$, thus improving the steric accessibility of the target DNA to the MB. Furthermore, this poly-A oligo also served to hybridise to the base of the molecular beacon, effectively increasing rigidity of this base, and forcing the MB to be extended away from the nanoparticle surface for enhanced access for target hybridisation.

As expected, increasing the Poly-T: MBs ratio in the immobilisation solution, resulted in an increase in the hybridization efficiency (Figure 2B) confirming that steric hindrance is a limiting factor for onAuNPs immobilised MBs (9). When the Poly-A sequence was added in the immobilisation solution, at equivalent molar ratios of the poly-T and MBs, a significant improvement in the hybridisation efficiency was recorded (Figure 2B). This was due not only to a reduction in steric hindrance, but also to the fact that the MBs are forced to extend as far as possible from the nanoparticle surface, resulting in improved hybridisation and a higher fluorescent signal.

\section{5. $D Q A 1 * 05 *$ and $D Q B 1 * 02 * M B$ s designs}

Once the surface chemistry and molecular beacon design had been optimised using the DQA $1 * 0201$ 
allele, MB probes were designed for the detection of the DQA $1 * 05 *$ and DQB $1 * 02 *$ alleles, using the IMGT/HLA database (39) and the Handbook of the International Hystocompatibility Working Group (40). Free online support (PerlPrimer (37), DNAmelt (41) and UNAfold (42)) were used to evaluate and refine the MB designs. The specificity of the designed MBs was evaluated by comparing the fluorescence response upon hybridisation with $100 \mathrm{nM}$ of the fully complementary target with those recorded in the case of the 1 base mismatched sequences (DQA1*0503 and DQB1*0203). Fluorescence measurements were recorded 20 minutes after target addition, and as can be seen in Figure 3A, the proposed MBs did not show very good specificity, particularly in the case of the $\mathrm{DQB} 1 * 02 *$. In order to overcome this limited specificity a second set of MB probes (DQA $105 \mathrm{~Pb}$ and DQB102Pb from Table 1) were designed. Exploiting an ARMS-type approach (25), a mismatch was introduced into the recognition of the $\mathrm{MB}$, which was anticipated to have the effect of markedly reducing the stability of the duplex between the $\mathrm{MB}$ and the sequence with one mismatch, as effectively this duplex would now have two mismatches. Optimisation of the location where the mismatch should be introduced was performed with the help of the PerlPrimers on-line software (37), where different bases were substituted at various positions of the original recognition sequence and the free energy $(\Delta \mathrm{G})$ of the different duplexes calculated.

For both the MBs the highest destabilisation was found to occur by the replacement of a thymine (T) with an adenine (A), as highlighted in bold in the MBs sequence reported in Table 1.

In Table 2 the values of the free energy calculated for the original design and for the best ARMS design are reported. As expected the introduction of a mismatch in the recognition sequence resulted in a reduction of the $\Delta \mathrm{G}$ (stability) of the duplex, and this reduction in stability was markedly more notable, particularly for the DQB $1 * 02 * \mathrm{MB}$.

\section{LOCATION FOR TABLE 2}

Once the new set of MB designs were defined, the specificity of each of the Au-MB was again examined, and as can be seen in Figure 3B there is an appreciable improvement in the specificity of the two MBs.

\section{LOCATION FOR FIGURE 3}

\subsection{Detection}

In the experiments for the simultaneousdetection of the two alleles, the AuNPs were functioanlised with a solution containing a molar ratio of 0.5:0.5:2:3 of DQA105Pb, DQB102Pb, poly-T spacer oligo, and the complementary poly-A oligo, respectively. The simultaneous presence of the two MBs onto 
the AuNPs was verified by DTT cleavage experiments and monitoring the fluorescence of the Rhodamine 6G and Atto $647 \mathrm{~N}$ labels. These measurements indicated an average loading of $22 \pm 2$ and of $21 \pm 2(\mathrm{n}=3)$ MBs for AuNP for the DQA105Pb and DQB102b MBs, respectively, loading values that are consistent with previous reports (9). Furthermore the total loading was consistent with those recorded in the case of the singleplex AuNP-MBs. The prepared AuNP-MBs conjugates were then used for the simultaneous detection of the two target alleles. This was performed following the same protocol used for the singleplex experiments with the only difference that in this case fluorescence measurements were performed sequentially using the wave lengths specific for each of the fluorophores (Figure 4). Clearly the proposed detection platform can selectively detect the desired targets, moreover no cross-talk between the two fluorophore was recorded, highlighted by the fact that the opening of one of the MBs did not induce any significant increase in the fluorescence due to the fluorophore associated with the other $\mathrm{MB}$, moreover no significant variation in the intensity of the fluorescence of each MBs was recorded when only one or both MBs were opened (hybridised with the fully complementary target).

\section{LOCATION OF FIGURE 4}

A closer look to the analytical performances of the multiple targets nano-particles based assay, prepared according to the optimised oligonucleotide layer composition and molecular beacon designs, was taken. In this specific experiments $\mathrm{DQB} 1 * 02$ targets have been used. The sensing platform prepared using $2.5 \mathrm{nM}$ starting concentration of the AuNPs showed a linear dynamic range between 0 and $10 \mathrm{nM}$ (Figure 5) with a limit of detection of ca. $0.5 \mathrm{nM}$ that is consistent with those previously reported for similar systems (12). To be notice that the linear dynamic range was narrower if compared with those recorded for Au-nanopaticle modified with a single molecular beacon (12); this was expected due to the reduced number of MB immobilised onto the Au nanoparticle surface.

\section{LOCATION FIGURE 5}

\section{Conclusion}

In the reported work the development of a multiple parameters fluorescence detection platform, based on molecular beacon functionalised gold nanoparticles, for the low resolution HLA typing of the coeliac disease associated DQ2 genotype was presented. The use of molecular beacon, having the recognition element partially incorporated into the stem resulted in a higher loading of the beacon onto the gold nanoparticle, presumably due to the reduced size of the loop, leading to a $40 \%$ increase in fluorescence signal. Thiolated molecular beacons with a 10-T appendage at the 3 ' end were coimmobilised with thiolated 10-T spacer oligos. An increase in fluorescence was observed due to the 
incorporation of the spacer oligos and was further improved upon addition of a 10-A oligo, which not only further spaced out the immobilised molecular beacons enhancing steric accessibility, but also gave the molecular beacon base rigidity thus extending the molecular beacon from the nanoparticle surface, rendering it more available for interaction with target DNA. Probes specific for the detection of the DQA $1 * 05 *$ and $\mathrm{DQB} 1 * 02 *$ alleles exploiting an ARMS-type approach consisting of introducing a mismatch facilitated the discrimination of a single point mismatch. The, gold nanoparticles bifunctionalised with the two molecular beacons, each labelled with a different fluorophore label, were demonstrated to selectively and simultaneously detect the DQB $1 * 02$ and DQA1*05 alleles achieving in this way the low resolution HLA-typing of the coeliac disease associated DQ2 genotype. The proposed platform was shown to have a limit of detection of ca. $0.5 \mathrm{nM}$ with a linear dynamic range between 0 and $10 \mathrm{nM}$.

\section{Acknowledgement}

This work was carried out with partial financial support from the Commission of the European Communities, specific RDT program "Coeliac Disease Management Monitoring and Diagnosis using Biosensors and an Integrated Chip System, CD-MEDICs, FP7-2007-ICT-1-216031". V.Beni acknowledges the European Union's Seventh Framework Program (FP7/2007-2013) under grant agreement no. PERG-GA-2009-256542 for financial support.

\section{References}

1. Green PHR, Jabri B. (2003) Lancet 362: 383-91

2. Ollikka P, Raussi HM, Laitala V, Jaakkola L, Hovinen J, et al. (2009) Analytical Biochemistry 386: 20-9

3. Monsuur AJ, de Bakker PIW, Zhernakova A, Pinto D, Verduijn W, et al. (2008) Plos One 3

4. HLA-Ready Gene Coelic Disease. INNO-TRAIN Diagnostik GmbH

5. EU GEN. Eurospital SpA

6. Louka AS, Sollid LM. (2003) Tissue Antigens 61: 105-17

7. Giljohann DA, Mirkin CA. (2009) Nature 462: 461-4

8. Li YS, Zhou XY, Ye DY. (2008) Biochemical and Biophysical Research Communications 373: 457-61

9. Song SP, Liang ZQ, Zhang J, Wang LH, Li GX, Fan CH. (2009) Angewandte ChemieInternational Edition 48: 8670-4

10. Drummond TG, Hill MG, Barton JK. (2003) Nature Biotechnology 21: 1192-9

11. Nasef H, Beni V, Ozalp VC, O'Sullivan CK. (2010) Analytical and Bioanalytical Chemistry 396: $2565-74$

12. Beni V, Hayes K, Lerga TM, O'Sullivan CK. (2010) Biosensors \& Bioelectronics 26: 307-13 
13. Tyagi S, Kramer FR. (1996) Nature Biotechnology 14: 303-8

14. Marras SAE, Kramer FR, Tyagi S. (1999) Genetic Analysis-Biomolecular Engineering 14: $151-6$

15. Tyagi S, Bratu DP, Kramer FR. (1998) Nature Biotechnology 16: 49-53

16. Wang H, Li JS, Wang YX, Jin JY, Yang RH, et al. (2010) Analytical Chemistry 82: 7684-90

17. Du H, Disney MD, Miller BL, Krauss TD. (2003) Journal of the American Chemical Society 125: 4012-3

18. Du H, Strohsahl CM, Camera J, Miller BL, Krauss TD. (2005) Journal of the American Chemical Society 127: 7932-40

19. Dubertret B, Calame M, Libchaber AJ. (2001) Nature Biotechnology 19: 365-70

20. Maxwell DJ, Taylor JR, Nie SM. (2002) Journal of the American Chemical Society 124: 9606-12

21. Mo ZH, Yang XC, Guo KP, Wen ZY. (2007) Analytical and Bioanalytical Chemistry 389: 493-7

22. Ray PC, Darbha GK, Ray A, Walker J, Hardy W. (2007) Plasmonics 2: 173-83

23. Ray PC, Fortner A, Darbha GK. (2006) Journal of Physical Chemistry B 110: 20745-8

24. Zhang SB, Wu ZS, Xie M, Shen GL, Yu RQ. (2009) Chinese Journal of Chemistry 27: 523-8

25. Wang WP, Zhang XD, Zhou GH. (2010) Molecular Biotechnology 44: 1-7

26. Wang KM, Tang ZW, Yang CYJ, Kim YM, Fang XH, et al. (2009) Angewandte ChemieInternational Edition 48: 856-70

27. Huang X, Qian W, El-Sayed IH, El-Sayed MA. (2007) Lasers in Surgery and Medicine 39: $747-53$

28. Yun CS, Javier A, Jennings T, Fisher M, Hira S, et al. (2005) Journal of the American Chemical Society 127: 3115-9

29. Chhabra R, Sharma J, Wang HN, Zou SL, Lin S, et al. (2009) Nanotechnology 20

30. Zhao WT, Lin L, Hsing IM. (2009) Bioconjugate Chemistry 20: 1218-22

31. Gonzalez-Galarza FF, Christmas S, Middleton D, Jones AR. (2010) Nucleic Acids Research 39: D913-D9

32. Tsourkas A, Behlke MA, Bao G. (2002) Nucleic Acids Research 30: 4208-15

33. Tsourkas A, Behlke MA, Rose SD, Bao G. (2003) Nucleic Acids Research 31: 1319-30

34. Grabar KC, Freeman RG, Hommer MB, Natan MJ. (1995) Analytical Chemistry 67: 735-43

35. Zu YB, Gao ZQ. (2009) Analytical Chemistry 81: 8523-8

36. Hurst SJ, Lytton-Jean AKR, Mirkin CA. (2006) Analytical Chemistry 78: 8313-8

37. Liu XO, Atwater M, Wang JH, Huo Q. (2007) Colloids and Surfaces B-Biointerfaces 58: 3-7

38. Stakenborg T, Peeters S, Reekmans G, Laureyn W, Jans H, et al. (2008) Journal of Nanoparticle Research 10: 143-52

39. IMGT/HLA Database. EMBL-EBI 
40. Hurley C. K., Fernandez-Vina M., Middleton D., Noreen H., Schmeckpeper B., et al. (2002) HLA 2004: Immunobiology of the Human MHC

The Proceedings of the 13th IHWC

41. Markham NR, Zuker M. (2005) Nucleic Acids Research 33: W577-W81

42. Kibbe WA. (2007) Nucleic Acids Research 35: W43-W6 


\section{Figures and Tables}

Scheme 1: Schematic description of the different possible allele's combinations associated with the DQ2 genotypes. In black are highlighted the alleles that have been proven to be associated to CD and in white the alleles most commonly associated to them.

Scheme 2: Pictorial representation of the working principle of the detection platform based on AuNPs MBs conjugate.

Figure 1: TEM images of the AuNPs prior to (A) and following (B) functionalisation with MBs. The red arrows highlight the capping layer thickness.

Figure 2: A) Evaluation of the stem position/design on MB loading (DTT cleavage bar) and performances (Hybridisation bar) of AuNP-MB. B) Hybridisation efficiency of AuNP-MB as a function of the composition of the oligonucleotides monolayer assembled onto the AuNPs. Hybridisation experiments performed using $100 \mathrm{nM}$ of fully complementary target in 1x SSC buffer (pH 7.4).

Figure 3: Evaluation of the specificity, between single base differing sequences, of the AuNP-MB conjugates obtained using conventional (A) and ARMS type (B) MBs designs. Hybridisation experiments performed using $100 \mathrm{nM}$ of fully complementary target in 1x SSC buffer (pH 7.4).

Figure 4: Simultaneous detection of DQA1*05 and DQB1*02 target alleles and their discrimination versus single nucleotide mismatched alleles (DQA1*0503 and DQB1*0203). Hybridisation experiments performed using $100 \mathrm{nM}$ of fully complementary target in 1x SSC buffer (pH 7.4).

Figure 5: Calibration curve for DQBQ02 probe specific (DQB1*02) and unspecific (DQB1*0203) targets using the multiple parameters integrated sensing platform prepared from a starting concentration of the AuNPs of $2.5 \mathrm{nM}$ and with a molar ratio of $(0.5+0.5): 2: 3$ of the MBs, poly-T spacer oligo, and the complementary poly-A oligo, respectively. Measurements were carried out in triplicate.

Table 1: List of the oligonucleotides used.

Table 2: Comparison of the $\Delta \mathrm{G}$, calculated using the PerlPrimers on-line software (37), and of the 
discrimination factors recorded using the conventional and ARMS inspired MB's designs. 


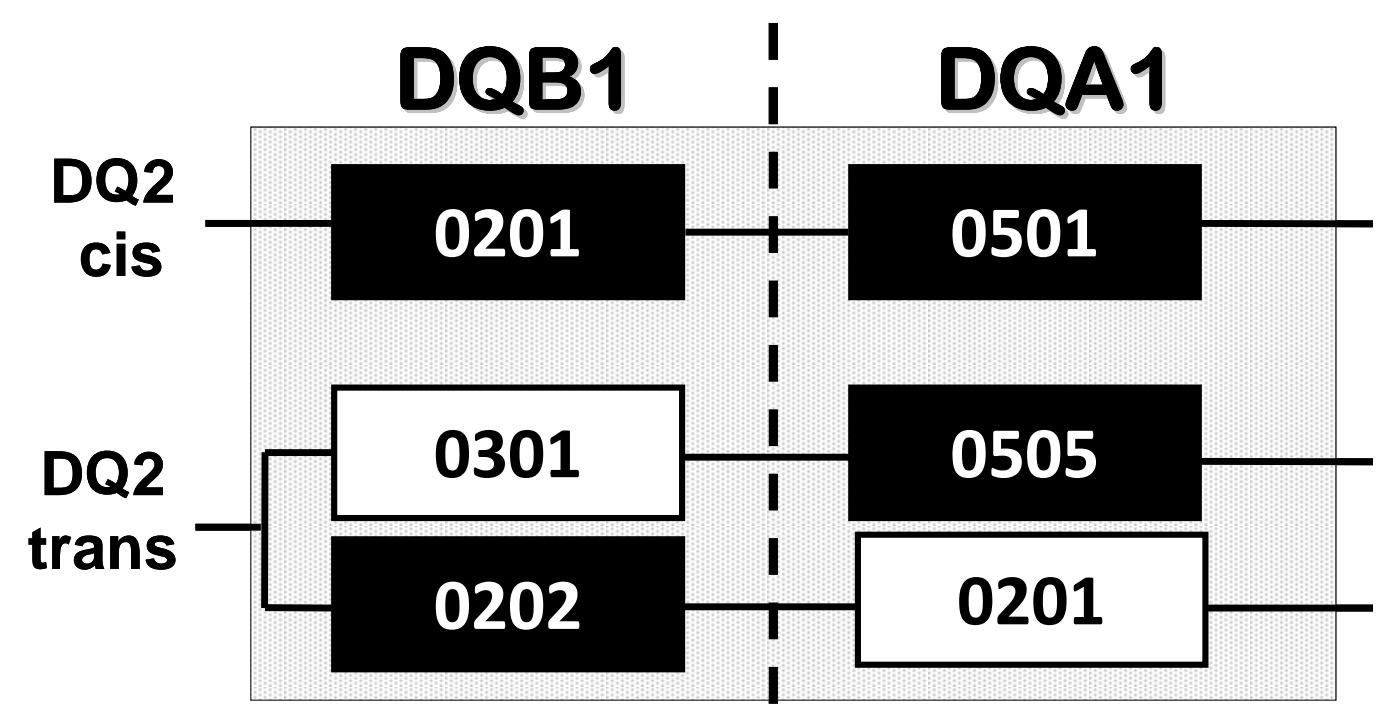

Scheme 1 


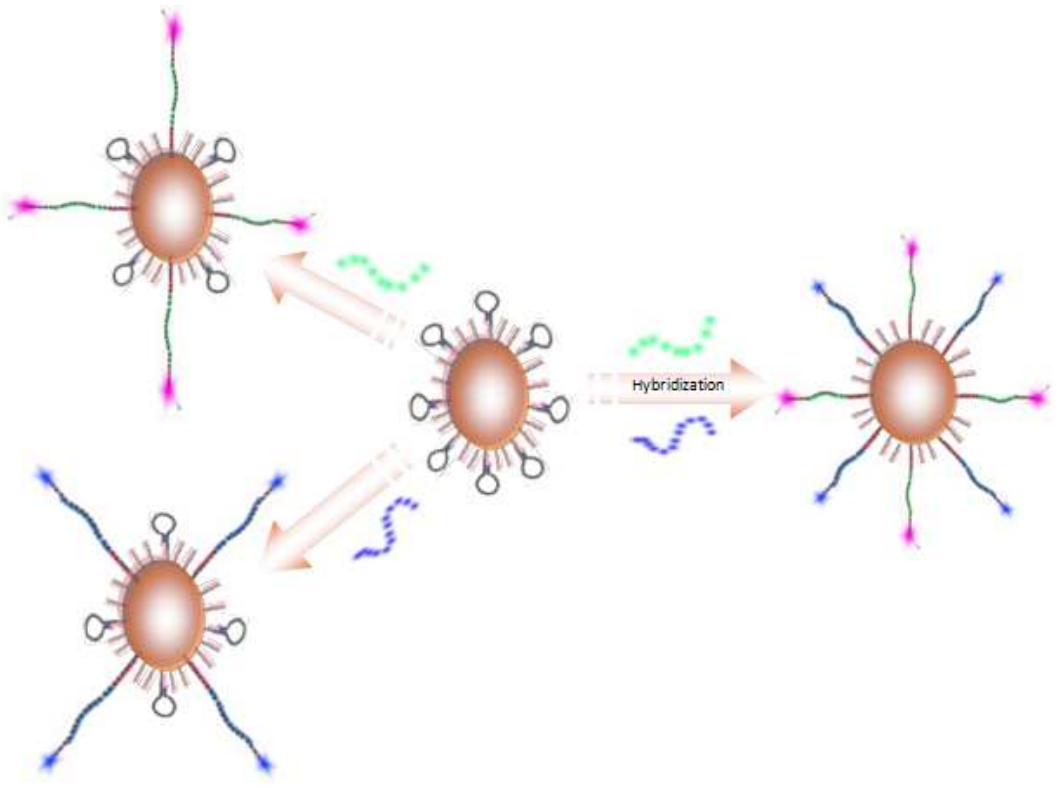

Scheme 2 

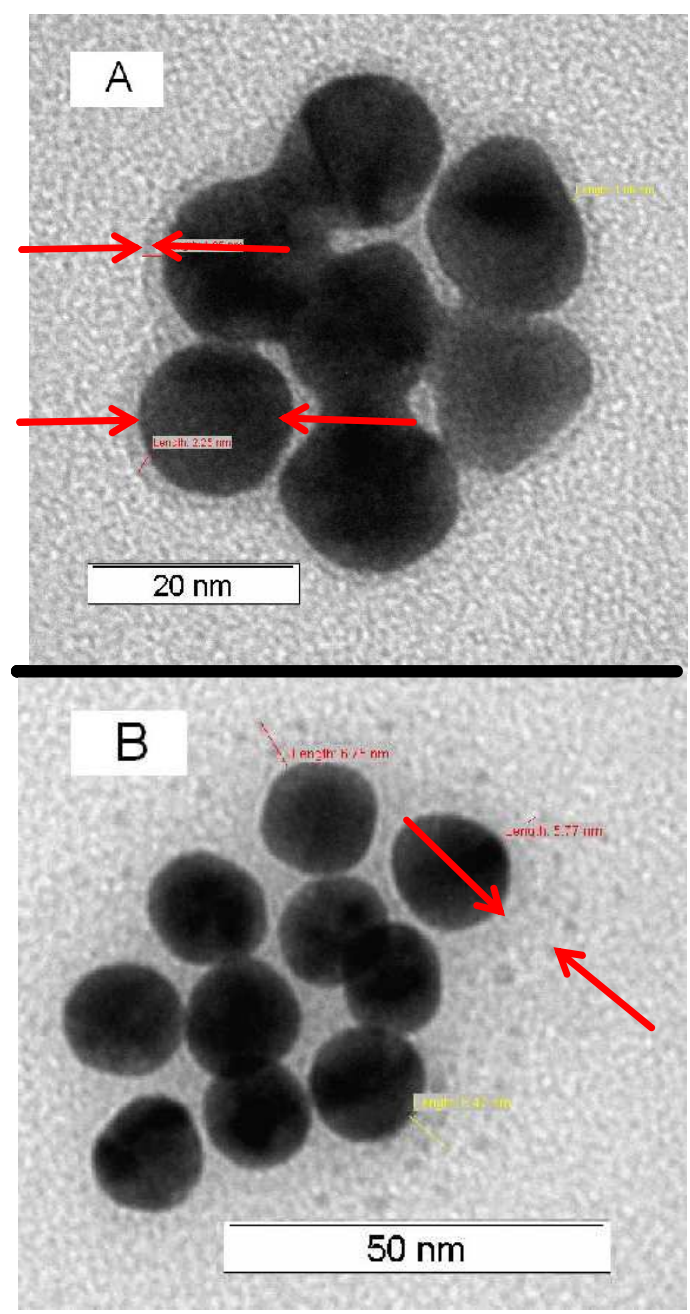

Figure 1 

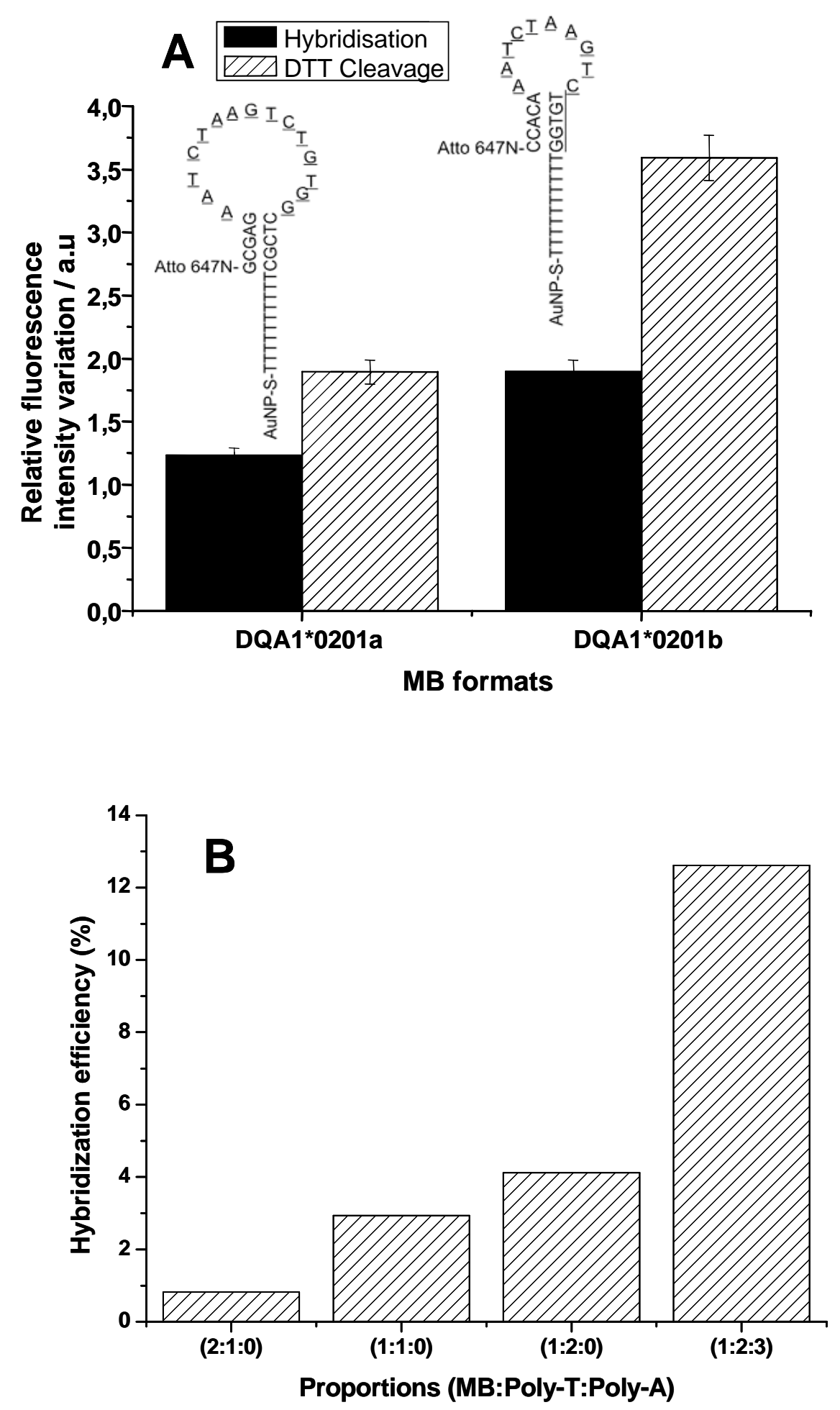

Figure 2 

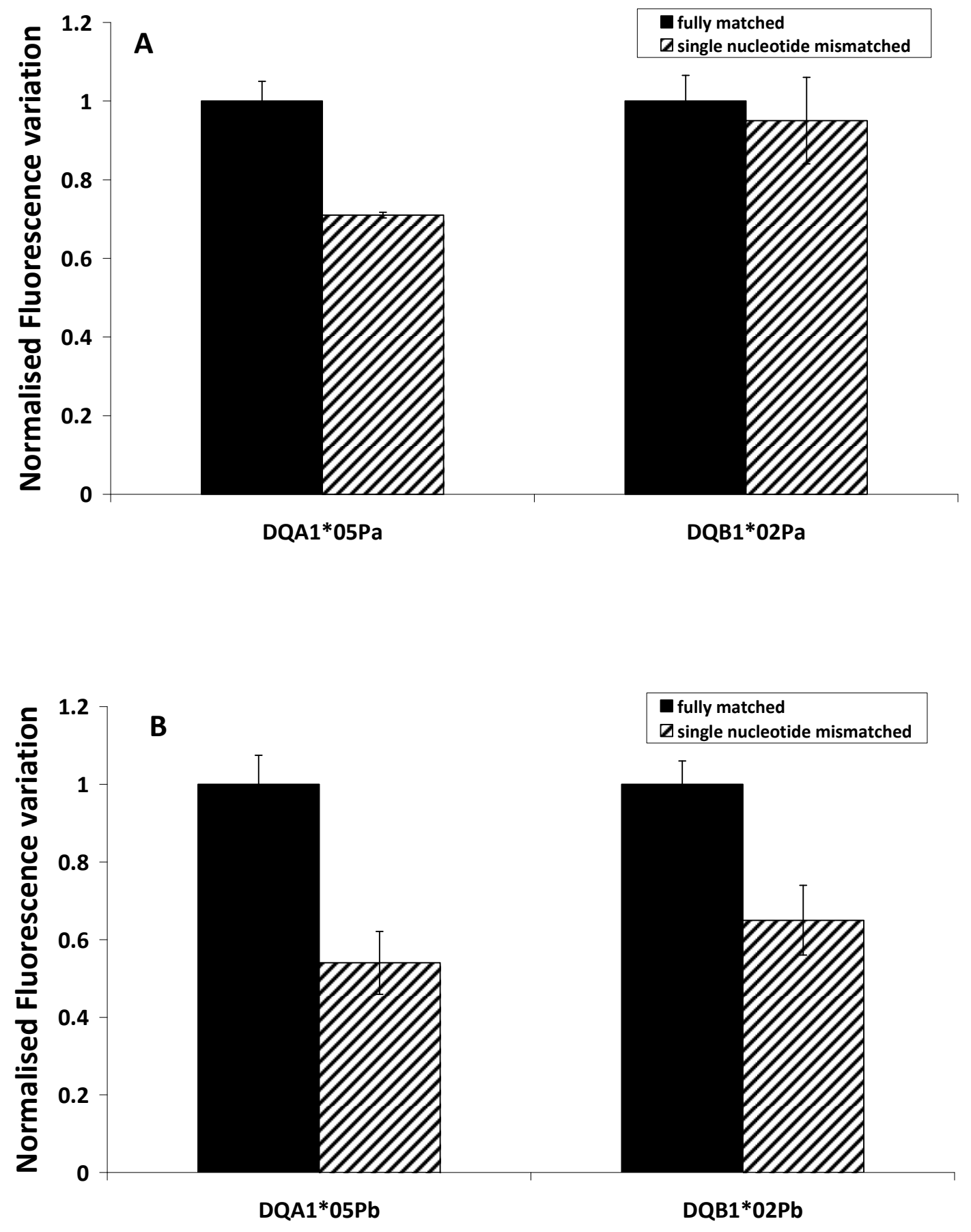

Figure 3 


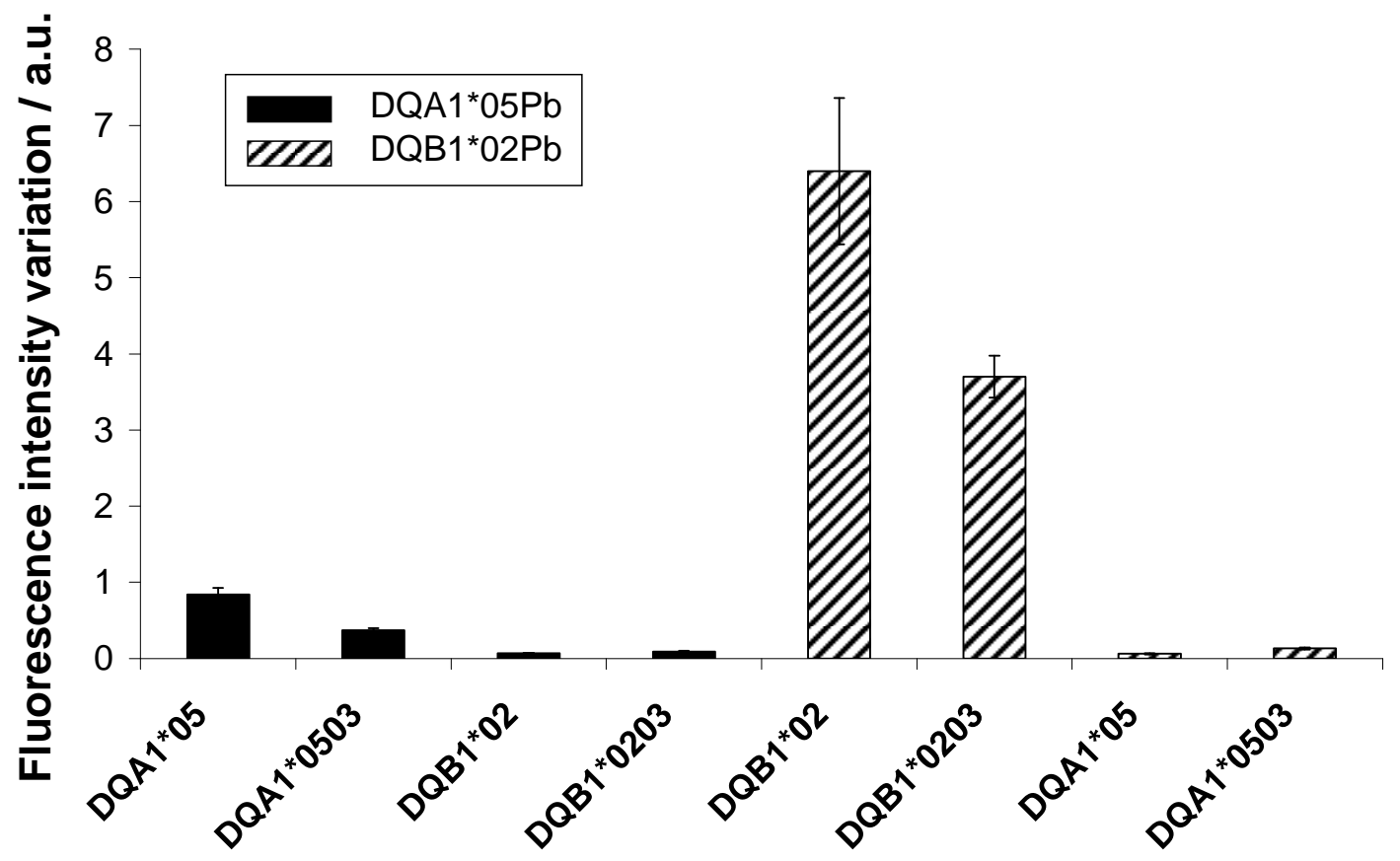

Figure 4 


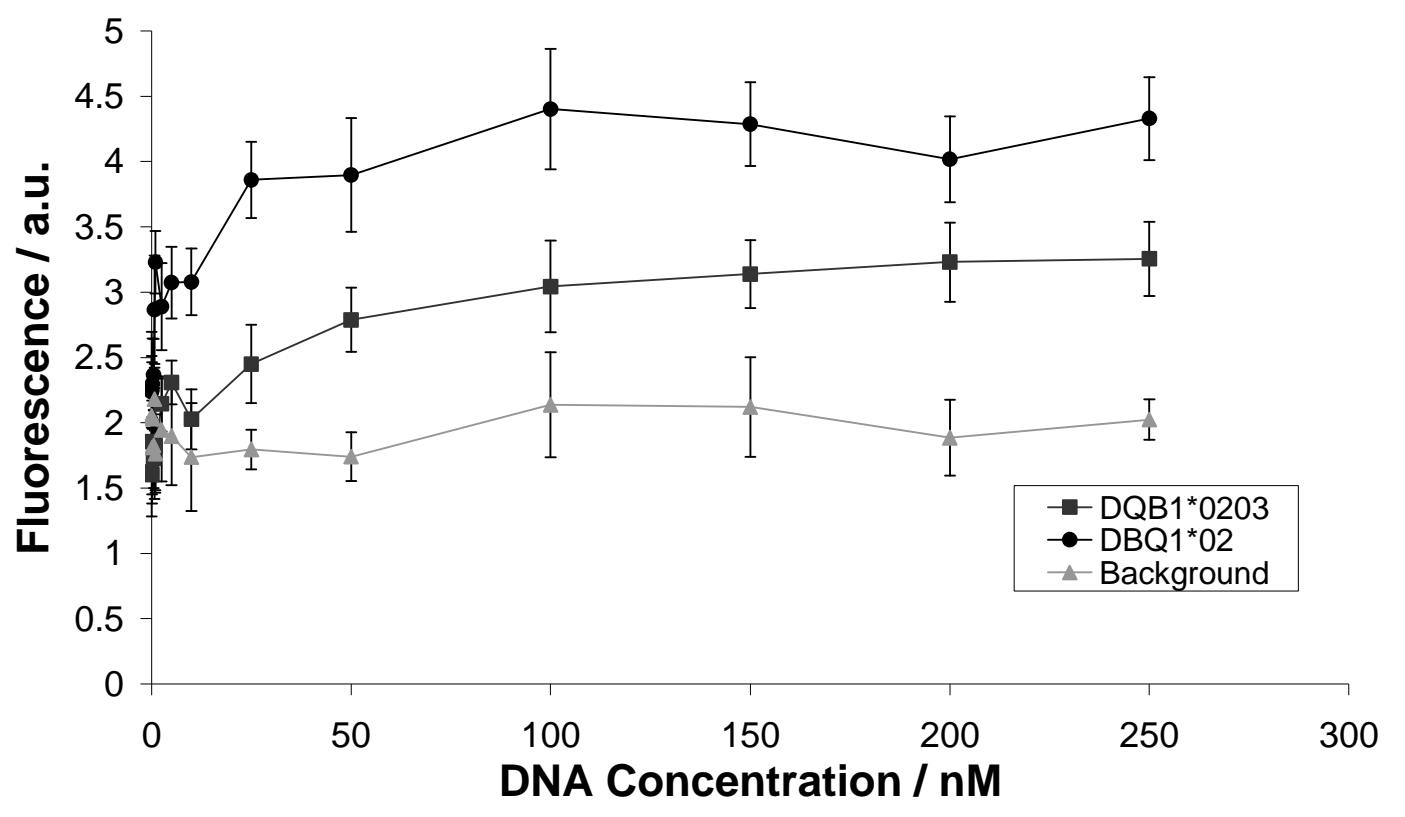

Figure 5 


\begin{tabular}{|c|c|}
\hline $\begin{array}{c}\text { Oligonucleotides } \\
\text { name }\end{array}$ & Sequences (5' - 3') \\
\hline \multicolumn{2}{|r|}{ Molecular beacons } \\
\hline DQA1*0201a & Atto $647 \mathrm{~N}-G C G A G$ AATCTAAGTCTGTGGCTCGC-(10T)-(CH2)3-SH \\
\hline DQA1*0201b & Atto $647 \mathrm{~N}-C C A C A$ AATCTAAGTCTGTGG-(10T)-(CH2)3-SH \\
\hline DQB1*02Pa & Atto $647 \mathrm{~N}-C T G C C T$ ACTCGGCGGCAGGCAG-(10T)-(CH2)3-SH \\
\hline $\mathrm{DQB} 1 * 02 \mathrm{~Pb}$ & Atto $647 \mathrm{~N}-C T G C C T$ ACTCGGAGGCAGGCAG-(10T)-(CH2)3-SH \\
\hline DQA1*05Pa & Rh6G-TCTGCT AACTCTCCTCAGCAGA-(10T)-(CH2)3-SH \\
\hline DQA $1 * 05 \mathrm{~Pb}$ & Rh6G-TCTGCT AACTCTCATCAGCAGA-(10T)-(CH2)3-SH \\
\hline \multicolumn{2}{|r|}{ "Spacing" and "Spacing complementary oligo" sequences } \\
\hline Oligo-T & TTTTTTTTTT-(CH2)3-SH \\
\hline Oligo-A & AAAAAAAAAA \\
\hline \multicolumn{2}{|r|}{ Targets } \\
\hline$\overline{\mathrm{DQA} 1} * 0201$ & CCACAGACTTAGATT \\
\hline $\mathrm{DQB} 1 * 02$ & CTGCCTGCCGCCGAGTA \\
\hline DQB1*0203 & CTGCCTGACGCCGAGTA \\
\hline DQA1*05 & TCTGCTGAGGAGAGTTA \\
\hline DQA1*0503 & TCTTCTGAGGAGAGTTA \\
\hline
\end{tabular}

Table 1 


\begin{tabular}{|c|c|c|c|c|}
\hline Target & \multicolumn{2}{|c|}{ Original MB designs } & \multicolumn{2}{|c|}{ ARMS inspired MB designs } \\
\hline & $\begin{array}{c}\Delta G \\
\left(k^{\prime} l a \mathrm{~mol}^{-1}\right)\end{array}$ & $\begin{array}{c}\text { Discrimination } \\
\text { factor }\end{array}$ & $\begin{array}{c}\Delta G \\
\left(k^{\prime} \text { cla } m o l^{-1}\right)\end{array}$ & $\begin{array}{c}\text { Discrimination } \\
\text { factor }\end{array}$ \\
\hline DQA1*05 & -23.7 & \multirow[t]{2}{*}{1.5} & -19.9 & \multirow[t]{2}{*}{2.3} \\
\hline DQA $1 * 0503$ & -17.7 & & -13.9 & \\
\hline DQB1*02 & -29.3 & \multirow[t]{2}{*}{1.1} & -24.4 & \multirow[t]{2}{*}{1.8} \\
\hline DQB $1 * 0203$ & -25.2 & & -17 & \\
\hline
\end{tabular}

Table 2 